\title{
Hacia Una Caracterización De La Producción Oral Espontánea De Los Estudiantes Universitarios Benineses De ELE
}

\author{
Laurent-Fidèle Sossouvi, (PhD) \\ Université d’Abomey-Calavi, Benín
}

doi: 10.19044/esj.2016.v12n35p209 URL:http://dx.doi.org/10.19044/esj.2016.v12n35p209

\begin{abstract}
This exploratory study analyzed the errors made by Beninese university students of Spanish as a Foreign Language $(n=100)$ in situations of spontaneous speech both inside and outside the classroom, in order to characterize them linguistically. The corpus contains 10 hours of high quality recorded spontaneous conversations or interactive communicative activities. The results indicate the main categories of interference of the available languages, heterogeneous sources of errors, highlighting the creativity and the dynamism of the participants' linguistic practices, showing a tendency to mix or switch the codes of their repertoire. Finally, possible pedagogical and curricular suggestions are explored.
\end{abstract}

Keywords: Multilingualism, crosslinguistic influence, oral interlanguage, Spanish as a foreign language, languages in contact

\section{Resumen}

En este estudio exploratorio se analizan los errores que cometen estudiantes universitarios benineses de ELE $(n=100)$ en situaciones de habla espontánea dentro y fuera del aula, con el fin de caracterizarlos lingüísticamente. Los datos pertenecen a un corpus de interlengua oral de diez horas de grabación, compuesto por actividades comunicativas interactivas extendidas en el tiempo. Los resultados señalan las principales categorías de interferencia de las lenguas disponibles, un conjunto heterogéneo y multiforme de motivos que originan los errores, poniendo de manifiesto la creatividad y el dinamismo de las prácticas lingüísticas de los participantes, una tendencia a mezclar o alternar los códigos de su repertorio. Finalmente, se exploran las posibles sugerencias a nivel pedagógico $\mathrm{y}$ curricular. 
Palabras Clave: Multilingüismo, influencia translingüística, interlengua oral, español como lengua extranjera, lenguas en contacto

\section{Introducción}

Desde el comienzo del siglo XX hasta hoy, la comunicación oral sigue teniendo toda su vigencia en el proceso de enseñanza-aprendizaje de una lengua extranjera (LE). Asimismo, la evolución de las metodologías hasta el Marco común europeo de referencia para las lenguas (MCER) y el enfoque orientado hacia la acción no dejan de aumentar su envergadura. Pero varios estudios anteriores (por ejemplo, Cenoz, Hufeisen y Jessner, 2001; De Angelis, 2007; Jessner y Cenoz, 2007; Jarvis, 2014, entre otros) han podido mostrar que la adquisición de la segunda lengua (L2) es distinta a la de la tercera lengua (L3), cuarta lengua (L4) o más lenguas adicionales. En efecto, el aprendiente de una L2 solo aprovecha las experiencias de adquisición de la primera lengua (L1), mientras que el aprendiente multilingüe que quiere aprender otra lengua adicional ya tiene las experiencias de todas estas lenguas, lo que constituye a su vez una ventaja en lo que se refiere a las estrategias y los conocimientos metalingüísticos. De este modo, la adquisición de una LE en un contexto monolingüe no sería lo mismo que en un contexto genuinamente multilingüe de Benín, un país pluricultural, multinacional y multiétnico de África occidental, donde varias lenguas (73 lenguas nacionales) de estatus diferentes cohabitan, y donde la mezcla y alternancia de códigos son muy frecuentes entre sus habitantes (Sossouvi, 2004; 2009a; 2009b; 2014).

En trabajos anteriores (Sossouvi, 2004; 2009a; 2009b) ya hemos analizado las producciones tanto escritas como orales de los aprendientes secundarios de Benín cuyos resultados se caracterizan por la mezcla de lenguas. El presente artículo pretende analizar algunos de los discursos espontáneos de estudiantes de grado, multilingües activos que aprenden español como lengua extranjera (ELE), precisamente, queremos intentar caracterizar lingüísticamente su interlengua oral, para saber si esta constituye una mezcla de códigos como la de los secundarios. Una correcta caracterización de estas producciones permitiría entender mejor los procesos psicológicos y lingüísticos internos que se activan en el proceso de aprendizaje de ELE. Es una realidad que el español está alcanzando un gran auge en el país (Sossouvi, 2014), y es interesante saber cómo los estudiantes universitarios se comunican en esta lengua, que solo se habla en aula, sus escollos principales, las características de sus interlenguas, con el fin de contribuir a agilizar y facilitar el uso de la lengua cervantina, así como proponer algunas líneas metodológicas para su enseñanza. Pretendemos aplicar la metodología del análisis de errores para describir y analizar los usos erróneos más frecuentes en los participantes. 
Así pues, para tratar esta posible caracterización, el presente estudio realizará primero un repaso por algunos aspectos teóricos, con el propósito de establecer las bases sobre las que se asentará la investigación. Luego se centrará en todos aquellos aspectos metodológicos y procedimentales que la sustentan. Posteriormente, se analizarán y discutirán los resultados obtenidos, con el propósito de dar cuenta de los hallazgos relevantes, establecer su importancia e interpretarlos. En última instancia, se realizará una recapitulación de todos estos aspectos para aplicar posibles líneas de investigación futura.

\section{Perspectiva Teórica \\ Concepto de interferencia}

La interferencia se refiere a las anomalías de la norma lingüística en la producción de los aprendientes que ya tienen conocimientos de otras lenguas. La razón es que las estructuras de una o de las lenguas del repertorio lingüístico del aprendiente (la L1, la L2 o la L3) se infiltran en la lengua meta adicional. En otras palabras, el aprendiente importa elementos de las estructuras de una lengua desde las estructuras de otra(s) lengua(s). Es un proceso que se observa muy a menudo en situación de lenguas en contacto, en el aula, en los contextos multilingües, etc. Efectivamente, el aprendiente de una nueva lengua no es ninguna tabla rasa; las estructuras de las lenguas de su repertorio lingüístico forman un género de pasador o colador por el cual transita el reciente material lingüístico. A la hora de aprender una nueva lengua, los aprendientes activan los recursos de las lenguas aprendidas con anterioridad, estas pueden servirles para confirmar la comprensión y aumentar sus destrezas en la lengua meta. En el mismo sentido, las lenguas del repertorio lingüístico del discente constituyen una herramienta que les ayuda a pensar y a entender las estructuras, el contenido tanto escrito como oral de la lengua meta. Estas lenguas constituyen ante todo una ayuda, y no un obstáculo para la adquisición de la nueva lengua. Además, la interferencia no es el único factor que origina el error y tampoco conduce siempre a una infracción de las normas del sistema, o a un desvío con respecto a una actuación esperada. En efecto, cuando dos lenguas tienen rasgos lingüísticos similares puede simplificar la adquisición de la lengua meta: es la transferencia positiva.

Los procesos de transferencia dependen del entorno, de la destreza, del estilo y del tipo de discente. Pueden producirse como una estrategia comunicativa consciente cuando el discente desconoce la forma correcta, la palabra acertada y, en caso de conocerla, por falta de automatización. Pueden deberse, asimismo, a la maleabilidad propia de la interlengua, a los conocimientos anteriores y a factores afectivos como el miedo a perder la identidad si se aprende bien la LE meta, o a la impresión de que la LE es una 
lengua desprestigiada o desvalorizada social y culturalmente. Están presentes en todos los niveles lingüísticos y las variables que pueden influir en su uso son la edad, la fase del aprendizaje, el modo de aprendizaje, los conocimientos anteriores del aprendiente, además de sus características individuales y cultura lingüística.

Según Jarvis y Pavlenko (2008), existen tres tipos de transferencia: la transferencia hacia adelante (forward transfer, de la L1 a una L2), la transferencia inversa o hacia atrás (reverse transfer, de la L2 a la L1) y la transferencia lateral (lateral transfer, a partir de la L2 a una L3), es decir, dos o más lenguas pueden influirse mutualmente de forma simultánea.

Por su parte, Ramón Torrijos (2009), en un trabajo cuya meta es estudiar el impacto de la influencia translingüística en el proceso del aprendizaje de la L2 concluye que, además de la mezcla de códigos, los factores sociales, las diferencias individuales, entre otros, la transferencia de la L1 desempeña un papel clave en la adquisición de la L2 y que debería constituir una preocupación para el profesorado de L2 o LE.

Karim y Nassaji (2013) tras repasar la literatura sobre los estudios de influencia translingüística consideran que la interferencia de la L1 en el aprendizaje de la L2 es un componente, una estrategia que ayuda a los aprendientes a producir, a desarrollar y a organizar su pensamiento.

\section{¿Qué se entiende por interlengua?}

La interlengua (IL) constituye una noción insustituible del proceso de aprendizaje de LEs y de sus distintas etapas. La hipótesis de la IL estriba en la idea según la cual el contacto de una L1 con una L2 o LE tiende a crear en los aprendientes un código particular, distinto de ambas lenguas. Este tercer código, basado en las hipótesis que los aprendientes elaboran respecto a la lengua meta, funciona según reglas propias y tiene su propia dinámica de transformación. Cualquiera que sea el nivel del discente este código intermedio se llama generalmente interlengua desde el trabajo de Selinker (1972), si bien ha recibido también otras denominaciones: “idioma idiosincrásico” Coder (1971); “sistema aproximado” (Nemser, 1971); "sistema intermedio” (Porquier y Frauenfelder, 1980: 32); "lengua del aprendiz” (James, 1990; Lightbown y Spada, 1993). Generalmente, el aprendiente es considerado más como un ser que produce un código peculiar a partir de su práctica y recursos intrínsecos que en función de lo mal que habla la LE, se fundamenta en la interacción de las propiedades estructurales y funcionales de la L1, de la LE y de todo el bagaje lingüístico previo del aprendiente, además de la Gramática Universal (cf. Dewaele, 1998; Jake, 1998; Fuller, 1999; Pavlenko y Jarvis, 2002; Herdina y Jessner, 2002, entre otros). La IL se singulariza por su sistematicidad, mutabilidad y permeabilidad, no obstante la afinidad con la LE no es directa, al 
reestructurarse cada vez que un rasgo flamante se mete en el sistema. De acuerdo con Ellis (2008: 968), la interlengua:

"has come to be used with different but related meanings: (i) to refer to the series of interlocking systems which characterize acquisition, (ii) to refer to the system that is observed at a single stage of development ("an interlanguage”), and (iii) to refer to particular L1/L2 combinations”.

\section{El estudio empírico}

\section{¿Quién ha participado en esta investigación?}

En este estudio se recogieron datos de dos grupos de estudiantes universitarios benineses $(n=100)$ de diferentes edades y con diferentes edades de inicio. Aprenden español en un contexto institucional en AbomeyCalavi y comenzaron a aprender esta LE como verdaderos principiantes en el Instituto de Educación Secundaria.

Un primer grupo estaba formado por 60 estudiantes de primer curso de Grado $\left(1^{\circ}\right)$, que tenían una edad comprendida entre los 17 años y los 22 años en el momento de la recogida de datos y pertenecían a tres niveles diferentes: aquellos que empezaron el aprendizaje del español a los 14 años en el tercer año de la Educación Secundaria (curso conocido como Quatrième), aquellos que lo iniciaron a los 16 años en Seconde, y aquellos que lo comenzaron a los 20 años en Terminale.

El segundo grupo constaba de 40 estudiantes de $2^{\circ}$ curso de Grado $\left(2^{\circ}\right)$ del mismo departamento, cuya edad oscilaba entre los 18 años y los 24 años en el momento de la recogida de datos.

Así, cabe destacar el dispar nivel de los participantes de este trabajo. Eran todos multilingües simultáneos y además del francés, que es la única lengua oficial, hablan las lenguas nacionales como el fongbe, el gungbe, el saxwegbe, el adjagbe, el gengbe, el aizogbe, el yoruba y las lenguas extranjeras como el inglés (iniciado desde el primer curso de la Educación secundaria e incluso desde la escuela primaria para algunos), el portugués, el latín y el chino (un semestre a cuatro semestre de curso en la universidad). Tenían poca posibilidad de comunicarse con hablantes nativos de esa LE, poca posibilidad para interactuar en ella, excepto en el contexto formal.

\section{Materiales y procedimientos utilizados}

Los datos recogidos para este trabajo provienen de diversas fuentes. En primer lugar, surgen de nuestras observaciones participantes como aprendientes y luego como docente en la Educación Secundaria y luego en la enseñanza superior. En segundo lugar, procedían de nuestras conversaciones extendidas en el tiempo desde 2012 hasta 2015, con los participantes dentro y fuera del aula. Nuestra meta era consolidar sus conocimientos, reforzar sus automatismos, ofrecerles la oportunidad de practicar la LE, capacitarlos para 
que puedan comunicarse en español en situaciones reales, darles la oportunidad de poner en práctica todo lo aprendido. Las conversaciones han sido recogidas diariamente mediante una grabadora MP4 a sabiendas de todos, así como con una cámara de vídeo Sony, con el fin de describir su habla real. La ficha de los errores y el diario docente nos sirvieron para apuntar o transcribir algunos enunciados erróneos. Este estudio longitudinal nos permitió refinar el análisis de los datos.

En horas de clase se administró a los estudiantes una serie de tareas o actividades orales comunicativas, tales como exposición de un tema seguido de debates, juegos comunicativos y juegos de rol, debates sobre un tema, actividades de comprensión oral, debate o conversación después del visionado de una película española o un podcast en español. Además, cabe señalar que el corpus oral que hemos seleccionado para la presente investigación ha sido escogido arbitraria y casualmente. Resulta conveniente aclarar que este trabajo basado en diez horas de grabación de nuestros corpus (y apuntes) se centró en identificar, describir, clasificar y explicar los errores espontáneos con mayor presencia en nuestro corpus de ELE (más de dos ocurrencias), sin tener en cuenta la evaluación de la gravedad de los mismos. Para respetar la confidencialidad y el anonimato de los participantes no pusimos ningún nombre, solo pusimos su curso. Por otro, considerando nuestros trabajos previos, no hemos optado por la errónea teoría del “culpable único” sino por la multicausalidad del origen de los errores.

\section{Síntesis de los resultados de la investigación}

A grandes rasgos, las interferencias producidas estaban relacionadas con los niveles básicos de la lengua, a saber, los niveles fónico, gramatical y léxico.

\section{Interferencia relativa a la pronunciación \\ Errores de articulación}

A. Errores relativos a consonantes

Errores debidos a la confusión de sonido

Resultan de procesos en que un sonido determinado se sustituye por otro.

a. Articulación [z] de $/ \theta /$

La grafía $\mathrm{z}$ corresponde en español a la consonante fricativa interdental $/ \theta /$, y en francés a la consonante sibilante sonora [z]. Por eso, algunos de nuestros participantes, acostumbrados al símbolo francés, cometieron errores en la pronunciación de su análogo español.

(1) “Benín es un país de paz [páz]” (1º).

(2) “... tiene luz [lúz] verde” $\left(1^{\circ}\right)$.

b. Pronunciación fricativa de $\mathrm{v}$ 
(3) "Soy feliz de verle [vérle]” $\left(1^{\circ}\right)$.

(4) "Hay que vivir [vivír] para luchar en la vida [vída]" $\left(2^{\circ}\right)$.

Estos fallos podrían deberse tanto a la interferencia del francés, de las lenguas locales del subgrupo gbe, como de una canción famosa de "Son by four”, "A puro dolor” que ha sido la música genérica de una serie que pasaba en la televisión nacional ORTB.

B. Errores relativos a vocales

c. Reducción de diptongos

(5) "Van a inogurar el nuevo anfiteatro" (1º); (cf. inaugurar).

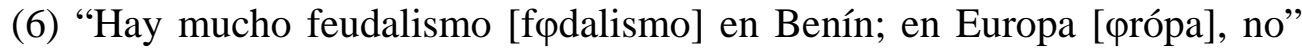
$\left(2^{\circ}\right)$.

En estos dos casos, no se tratan de la elisión de un sonido, sino que resultan de la aplicación de la solución francesa a secuencias de vocales que corresponden a diptongos decrecientes en español. Dicha solución resulta favorecida por la identidad gráfica de esas secuencias en francés y en español.

\section{Errores de acentuación}

a. Acentuación final, debida a la influencia del francés, donde el acento cae siempre en la última sílaba de la palabra, como en los ejemplos siguientes:

(7) "El problemá de la maltretancé" $\left(1^{\circ}\right)$; (cf. problema, maltrato).

(8) "Ha sido demostradá [demostradá] que..." (2º); (cf. Demostrada).

b. Pronunciación llana de palabras esdrújulas, explicable por la generalización de la regla de las palabras llanas.

(9) "Es mejor plantar arboles [arbóles] en junio" ( $\left.2^{\circ}\right)$; (cf. árboles).

(10) "Los ultimos [ultímos] serán los primerós” (1º); (últimos, primeros).

\section{Nivel gramatical}

En este apartado, solo nos proponemos presentar algunos aspectos de la gramática, en los planos de la morfología y sintaxis, dejando los aspectos del léxico para otro apartado.

Uso de los determinativos

Adición superflua o uso innecesario del determinativo artículo, resultante de la traducción literal del equivalente francés.

(11) "Por la primera vez, me he sentido maestro de mis decisiones..." $\left(2^{\circ}\right)$; (francés pour la première fois; cf. por primera vez).

Elección errónea

a. Del determinativo artículo

(12) “El pared está pintado en blanco" (1º); (francés le mur; cf. la pared).

(13) "El diabete es una enfermedad de los ricos” $\left(2^{\circ}\right)$; (francés le diabète; cf. la diabetes). 
Estas incorrecciones se deben a la imposición del género de la palabra francesa por asociación.

b. De los determinativos indefinidos.

(14) "Alguien de vosotros puede..." (1 $\left.1^{\circ}\right)$; (cf. Algunos de vosotros).

(15) “Nadie de vosotros sabe la respuesta correcta” (2); (cf. Ninguno de vosotros).

Estos participantes desconocen que no se admiten estructuras partitivas con "alguien" o "nadie".

Usos de las preposiciones

a. Omisión de la preposición de los complementos directos de persona (16) y (17) o sustantivos personificados (18), debida a la influencia del francés y de las lenguas locales que no exigen una marca especial ante el complemento directo.

(16) “Buscamos $\Phi$ mi prima en vano” (1º); (cf. a mi prima).

(17) "He visto $\Phi$ Julio esta mañana” ( $\left.2^{\circ}\right)$; (cf. a Julio).

(18) “No temen $\Phi$ la muerte” ( $\left.2^{\circ}\right)$; (cf. no temen a la muerte).

b. Elección errónea en galicismos sintácticos

(19) “Utilizamos cocina a gas” (1º); (francés cuisine à gaz; cf. cocina de gas).

c. Adición superflua de preposición, explicable por un mecanismo de generalización.

(20) “Se llamaba a Cecilia y tenía 18 años” (2º); (cf. se llamaba Cecilia).

Errores referentes a las concordancias

a. Concordancia de género

(21) "Hoy hace una calor sofocante” (1º); (francés une chaleur; cf. un calor).

(22) “El señor presidente está implicado en esta asunto” (2º); (francés cette affaire; cf. este asunto).

Estos casos resultan de la imposición del género de la palabra francesa por asociación. adjetivos.

Excepto los determinantes, hay errores en que están implicados los

(23) "Soy una chica alegra”" (10); (cf. alegre)

(24) “La vida comuna es fácil y difícil” (2º) (cf. común).

Estos errores resultan del uso de una única forma del adjetivo o de proceso de regularización.

a. Concordancia de número

Errores en que están implicados los sustantivos.

(25) “Los esprayes para matar mosquitos” (2º); (cf. esprays, espráis; aerosoles)

(26) “Hoy en día no se puede hacer nada sin las tiques” ( $2^{\circ}$ ); (cf. las tics). Estos fallos pueden atribuirse a la generalización de la formación del plural.

b. Concordancia de persona gramatical 
Uso de la tercera persona del plural con un sujeto singular, achacable a una concordancia basada en la semántica de los nombres colectivos (27) o utilización errónea de la tercera persona del plural por asociación a su equivalente en francés (28) y concordancia errónea de "haber" (como impersonal) (29).

(27) "la gente critican mucho" (10); (cf. critica).

(28). "No sé cuántas gentes vendrán hoy” (2º); (cf. no sé cuánta gente vendrá).

(29) “Habían allí muchos estudiantes esperando” (2º); (cf. Había allí...).

\section{Errores referentes al uso de los verbos}

Elección errónea de los tiempos

a. Presente de indicativo en lugar del pretérito perfecto

(30) “Después de comer nos somos descansados bien” ( $1^{\circ}$ ); (francés nous nous sommes bien reposés; cf. hemos descansado").

b. Usos incorrectos del imperativo en lugar de las formas de subjuntivo (31) "No hablad más de este" (1º); (cf. No habléis más).

(32) "No comed en clase” (2); (cf. No comáis).

Puede ser que olviden que en español no existe el imperativo en enunciados negativos, o una influencia de la negación en el imperativo francés e incluso de las lenguas nacionales como el fongbe.

Cambio de modo

Uso incorrecto del indicativo en lugar del subjuntivo

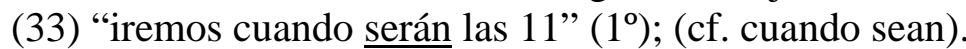

(34) "Llámeme cuando usted querrá" ( $2^{\circ}$ ); (cf. cuando quiera).

(35) “... para que la mujer africana trabaja” $\left(2^{\circ}\right)$; (cf. trabaje)

Estos casos reflejan la influencia del francés, sobre todo el (35) refleja la influencia del patrón del verbo equivalente en francés, cuyas formas de presente de indicativo y subjuntivo son parecidas (travaille). Puede ser que el presente de indicativo sea la forma fuerte, la forma verbal más conocida y usada.

Otros casos

a. Construcción pronominal de verbos no pronominales (36) y el caso contrario $(37,38)$ por asociación al verbo equivalente en francés.

(36) "No me recuerdo el nombre del protagonista" $\left(1^{\circ}\right)$; (francés se souvenir; cf. no recuerdo).

(37) "Los otros compañeros se descansan en su dormitorio" (2); (francés se reposer, cf. descansan).

(38) "Mudé de la casa de mi tío ayer" $\left(2^{\circ}\right)$; (francés déménager, cf. me mudé). 


\section{Interferencia pragmática}

(39) "Ven a comer" $\left(1^{\circ}\right)$; (cf. Te invito a comer o quiero compartir mi comida contigo).

Es un calco de las lenguas autóctonas como el fongbe (wa mi du nu).

(40) Un compañero estornuda: “A tus deseos” (1º); (cf. ¡Jesús! o Salud).

Puede ser un calco del francés "À tes souhaits").

(41) El profesor estornuda: "Bendición para usted” (2º); (cf. ¡Jesús! o Salud). Parece ser un calco del inglés (Bless you).

(42) “¿Usted ha despertado bien?” (1º); (cf. ¿Cómo está usted?).

Es un calco de las lenguas locales como el fongbe (Mi fon gandji (dagbe) a), el saxwègbe (mifón dagbe).

En todos estos casos se observa que los participantes han aplicado al español las hipótesis y los modelos de conducta que rigen en las lenguas previamente aprendidas.

\section{Interferencia conceptual}

Si por interferencia conceptual se entiende la influencia de las formas de presentación de conceptos de las lenguas previamente adquiridas en la producción en la lengua española, hemos registrado algunos casos. (43) "Los niños colocados al lado de alguien son como esclavos..." (20); (cf. niños adoptados). Es un calco del concepto vidomégon (fongbe) o "enfants placés" (francés).

(44) "Me gusta ver el tamtam (tambor) de los egungun" (2º); (cf. En Benín y Nigeria, es el espíritu de un antepasado difunto que ha regresado a la tierra).

(45) "Ha hecho zindo a su mujer" ( $2^{\circ}$ ); (fongbe; cf. ayuda mutua y solidaridad durante los funerales de los padres).

\section{Interferencia al nivel léxico}

a. Falsos amigos

La mayoría de las palabras implicadas son francesas y son casi similares a las formas españolas cuyo uso, inducido por esta semejanza, resulta erróneo.

(46) "Mi hermana acaba de acostar de una hija" (10); (francés accoucher d’un garçon; cf. dar a luz un niño).

(47) "Un niño afamado no puede aprender bien” (2º); (francés affamé, cf. Un niño hambriento).

(48) "Estoy fiero de mi padre" $\left(1^{\circ}\right)$; (francés fier; cf. Estoy orgulloso de mi familia).

b. Expresiones formal y/o semánticamente próximas

Formas híbridas

(49) "las lenguas etranjeras ..... la objectiva de los parentes..." (10); (cf. extranjeras, objetivo, padres). 
(50) “es un fiestardo” (2º); (francés fêtard; cf. fiestero, juerguista). Traducción literal del francés

(51) “Voy a dar un caso de figura” (2º); (francés cas de figure; cf. ejemplo).

(52) "Es simple como buenos días" ( $\left.2^{\circ}\right)$; (francés c'est simple comme bonjour; cf. es muy simple, está chupado).

(53) “Las asignaturas de tronco común” ( $2^{\circ}$ ); (francés tronc commun; cf. asignaturas troncales).

\section{Interferencias de otras lenguas}

\section{Interferencias de las lenguas locales}

Las interferencias al nivel léxico predominan.

a. Formas híbridas

(54) “Es un sociólogo y boólogo” ( $2^{\circ}$ ) (fongbe boó; cf. magia, grisgrís, una práctica esotérica, la potencia de las hojas).

b. Traducciones literales

(55) “Buena sentada, señor” (1º); (cf. ¡Qué tal!). Parece un calco de las lenguas locales fongbe (kudo aidjidjon), yoruba ( $\varepsilon$ ku kalk), e incluso del francés beninés informal (Bonne assise).

(56) “Buena llegada” ( $2^{\circ}$ ); (cf. Bienvenido; te damos la bienvenida). Puede ser la influencia del francés local beninés “Bonne arrivée” que es una influencia de las lenguas autóctonas fongbe ( $M i$ ka bo), yoruba (e ka ro), del gengbe (Mia we zon), etc.

(57) “El caca de la oreja” ( $\left.2^{\circ}\right)$; (fongbe tomi; cf. la cera o cerumen).

Estructuras que incluyen vocablos de las lenguas locales

(58) “voy a mi pueblo para las fiestas de xwetanu” ( $2^{\circ}$ ); (fongbe; cf. más o menos ceremonias consuetudinarias).

(59) "La mujer que acaba de dar a luz debe comer amanvive gnignan y lio para tener mucha leche” $\left(2^{\circ}\right)$. (fongbe; cf. salsa de verdura amarga (vernonia amygdalina) y una pasta ácida a base de maíz).

(60) "Hay muchos políticos kpayos" (2º); (fongbe; cf. de pacotilla, de poca calidad).

\section{Interferencias del inglés}

a. Traducciones literales

(61) “Soy 18 años y vengo de...” (1º); (inglés I’am 18 years old; cf. Tengo 18 años).

(62) ¿Por qué hay acento en las capitales letras? (2º); (inglés capital letters; cf. tilde en las mayúsculas).

b. Estructuras que incluyen vocablos ingleses

(63) “For eso, las matemáticas es por los scientifics...” (1); (cf. Por eso, científicos) 
(64) “He intentado varias veces upgrade windows 10 en vano" (2); (cf. actualizar a)

\section{Interferencia del portugués}

Solo tiene que ver con estructuras que incluyen vocablos portugueses.

(65) “El hijo de mi primo tiene dois años” (1 $\left.{ }^{\circ}\right)$; (cf. Dos años)

(66) “El aeroporto de Cotonú es pequeño” ( $\left.2^{\circ}\right)$; (cf. aeropuerto)

(67) "Hay ratos en la sala y ela tiene miedo" ( $\left.2^{\circ}\right)$; (cf. ratas, ella).

Un último aspecto a tener en cuenta en esta exposición de resultados es que a medida que el participante avanza en sus competencias en la lengua cervantina, esa influencia de las distintas lenguas implicadas disminuye.

\section{Interpretación de los resultados}

El objetivo de este estudio fue analizar las interlenguas orales de estudiantes universitarios benineses y comprobar si se puede caracterizarlas como una mezcla de códigos, al igual que las del estudiantado secundario. El análisis de los datos indica que, al igual que las producciones de los secundarios, sus interlenguas orales pueden caracterizarse como mezclas de códigos. Evidencia que los participantes hacen uso de todos sus recursos, combinando distintas lenguas de su repertorio con el castellano, difuminando las fronteras entre las lenguas, movilizando a voluntad las similitudes fonéticas, morfológicas, léxicos y semánticas entre las lenguas disponibles, sobre todo el francés, por su proximidad y por ser lengua romance como el castellano. Este hecho puede ocurrir porque quienes aprenden una LE, estratégicamente hacen correlaciones y asociaciones entre las lenguas de su repertorio y la lengua meta, como lo indicaron anteriores investigaciones (Singleton, 1999; Cenoz, 2003; Odlin y Jarvis, 2004; Sossouvi, 2004; Pennycook, 2010; Jarvis, 2014; González Alonso, Villegas y García Mayo, 2016) que apuntaron que la distancia lingüística existente entre las lenguas disponibles constituía dato o factor estratégico en la influencia translingüística. Pero como se puede observar en muchos casos documentados, la similitud entre lenguas no solo facilita la adquisición de una LE, sino que también da pie a inconsistencias cuando no existan paralelismos exactos entre las lenguas en cuestión. Eso significa que el aprendiente debería adaptar los sistemas de las lenguas previamente adquiridas a los de la lengua meta, sobre todo adaptar elementos o estructuras existentes en las primeras que tuvieran una correspondencia en la LE, y viceversa.

Se ve que los participantes no son muy conscientes de las diferencias entre el francés y el español; consideran al primero el representante de las lenguas romances, sobre todo desde el punto de vista gramatical y léxico (incluso ortográfico). En efecto, el francés es la primera lengua en la que han 
aprendido a elaborar el razonamiento gramatical. En otras palabras, perciben el español como una variante dialectal del francés. Además, las lenguas nacionales beninesas, por ejemplo del subgrupo gbe, presentan entre ellas un grado elevado de intercomprensión. Una posible explicación es que para ellos, las lenguas forman parte de un repertorio al que se accede por sus propósitos comunicativos; las lenguas son indiscretas, convergentes y forman un sistema armonioso. Esta competencia multilingüe brota de la cultura o prácticas lingüísticas del país donde se comunican en varias lenguas a la vez, donde se activa todo el bagaje lingüístico previo para adquirir nuevas lenguas, donde las fronteras entre las lenguas se difuminan en cada nuevo proceso adquisitivo.

Las causas que originan los errores son heterogéneas y variopintas, si bien destaca la notable y omnipresente interferencia de la lengua de Molière y, con mucha menor incidencia, la de las lenguas locales, del inglés y del portugués. Los participantes se encuentran en situaciones donde la presión comunicativa excede su conocimiento de la lengua cervantina. De todos modos los errores registrados derivan de procesos múltiples que se asocian tanto a la idea de déficit (fonológico, gramatical, léxico) que subyace al concepto clásico de interlengua como a una estrategia cuya meta es lograr el mayor rendimiento en términos de intercomunicación, a la eventualidad de acceder a dos o más códigos en un contexto concreto de intercambio. En consecuencia, la descripción que proponemos de las producciones orales de nuestros participantes sugiere la necesidad de actualizar el concepto de interlengua tal y como se suele usar en los estudios sobre la adquisición de LEs, lo que, a su vez, tiene implicaciones teóricas y psicopedagógicas que intentaremos abordar en trabajos venideros.

Las producciones orales de nuestros participantes que se pueden caracterizar como erróneas responden parcialmente a las descripciones que han recibido los conceptos de error y de interlengua. En términos más específico, solo algunas de ellas resultan de procesos de interferencia en los que el estudiante recurre a una forma o estructura típica de la lengua francesa, de las otras lenguas previamente aprendidas en lugar del equivalente en español, sea por desconocimiento o a causa de un error puntual. Los otros casos, bastante considerables, sugieren que los procesos implicados conllevan una complejidad mayor de la que cabe asociar a la mera interferencia del francés y de las demás lenguas de su repertorio. Es lo que sucede, por ejemplo, con los numerosos casos de traducción y calco, así como con las abundantes formas híbridas que caracterizan las producciones de nuestros informantes.

Sus producciones orales no se pueden caracterizar solamente en términos de interferencia de las lenguas que forman parte de sus repertorios respectivos de acuerdo con la actitud de los propios informantes y con el 
contexto en que acaece la comunicación, cabe asociar a la lengua cervantina antes que a cualquiera de las otras lenguas habladas o conocidas por ellos. Tampoco en caso de interferencia, queda limitado el proceso a la simple interferencia de formas o expresiones francesas o de las demás lenguas conocidas al español. El análisis minucioso de las producciones indica que resultan evidentemente de procesos muy diversos que convergen en lo que se puede caracterizar como la combinación y la interacción de las lenguas implicadas en el proceso de aprendizaje. El contorno de mezcla que las caracteriza constituye el efecto conjunto de hechos aislados de alcance más o menos circunscrito, que remiten en general a lo que investigadores como Field (2002) han asociado al proceso de préstamo, concebido en términos de convergencia o mezcla de códigos desde el punto de vista tanto léxico como estructural, en otras palabras, al proceso de copiado por medio del cual una expresión o estructura perteneciente a una lengua determinada es adoptada por otra e integrada en ella (cf. Field, 2002: cap. 2-127). Un resultado similar encontramos en Pennycook (2010) cuyos participantes mezclaron códigos en hip hop para exhibir nuevas subjetividades e imágenes.

La primera característica de las producciones orales de nuestros participantes da cuenta de cuantiosos datos que se refieren a procesos interpretados en términos de préstamos o de inserción por algunos especialistas como Muysken (2000), Field (2002), Clyne (2003), entre otros. Efectivamente, a diferencia de los casos de inserción de formas francesas inalteradas o de las demás lenguas, que se pueden relacionar con la alternancia de códigos (cf. Field, 2002: 11-22), esos datos se caracterizan porque están integrados en la lengua española, si bien el grado y modo de integración puede variar de un componente de la lengua a otro, así como de una forma o expresión a otra y, para una misma forma, de una persona a otra. El proceso de integración que indica mayor complejidad tiene que ver con la adopción de los préstamos léxicos, y es el resultado de su adaptación a la fonología, la morfología, la sintaxis, la semántica y el léxico de la lengua que los acoge.

Como se puede observar en la sección anterior, un número considerable de los procesos que caracterizan las producciones de nuestros participantes tienen que ver con la interacción del español y el francés, así como las demás lenguas de su repertorio, en el sentido de que los informantes tienden a mezclar y combinar las lenguas de su repertorio lingüístico. La tendencia a la mezcla y combinación se nota en todos los niveles $\mathrm{y}$, globalmente, esos procesos perfilan esas producciones. Cabe asociar dicho perfil o acento global a la lengua cervantina, en el sentido de que las expresiones que conforman los enunciados proferidos por nuestros participantes siguen los patrones estructurales típicos del español o bien rellenan por medio de léxico español estructuras sintagmáticas típicas del 
francés o de las demás lenguas de su repertorio, con resultados a menudo distantes de la lengua meta.

En resumen, es de destacar que este efecto de mezcla y combinación no solo se nota en el marco de cada uno de los niveles del lenguaje, sino también entre diferentes niveles. Consideramos por tanto que descripciones de procesos asociados al contacto de lenguas, tales como las ofrecidas en estudios anteriores (Myers-Scotton y Jake, 1995, 2000; Jake y MyersScotton, 1997; Jake, 1998; Fuller, 1999; Muysken, 2000; Major, 2001; Pavlenko y Jarvis, 2002; Field, 2002) son parciales por definición, ya que el centrarse en uno o dos componentes de la lengua, no pueden pintar el perfil global característico de las producciones lingüísticas asociadas a este tipo de contexto.

Otra posible causa de esta estrategia de mezcla de códigos puede ser la cultura lingüística del país donde se tolera el uso de todos los medios o estrategias para hacerse comprender mejor o hacer pasar mejor el mensaje, $\mathrm{y}$ donde se utilizan todos los recursos lingüísticos servibles para llegar a un rendimiento óptimo y eficiente de intercambio, sobre todo en contextos distendidos e informales. Las variedades y variaciones de lenguas, las alternancias y mezclas de códigos han sido o son percibidas siempre como una bendición. También es posible que esta mezcla de códigos haya sido inducida por el desconocimiento de las normas o valores socioculturales de la lengua cervantina, el anhelado multilingüismo, la intención de evidenciar la identidad lingüística en las interacciones en la lengua meta.

La heterogeneidad de niveles lingüísticos en ELE observada en nuestros participantes es coherente con estudios previos (Buck y McAllister, 2001) que mostraron que el estudiantado de Grado en LE estaban, en su mayoría, por debajo del nivel aceptable. Los errores pragmáticos e interculturales registrados en nuestro corpus son similares a los encontrados en diversos estudios (Kiuchukova-Petrinska, 2010; Su, 2012) explicables por las diferencias culturales y la falta o el descuido de la competencia sociocultural e intercultural en el currículo académico.

Con todo, conviene no perder de vista que estamos en un contexto formal, y que desde una perspectiva didáctica o metodológica, los estudiantes necesitan una formación sociolingüística, cultural y pragmática del español que le permitan interactuar en todas las situaciones comunicativas. Además, los datos invitan a reflexionar sobre la necesidad de adecuar las metodologías docentes a las nuevas demandas del siglo XXI, a utilizar en el aula de ELE en el país materiales diversos y atractivos que favorecen y fomentan la competencia comunicativa, que aseguran un aprendizaje significativo. El diseño curricular, los materiales y recursos didácticos deberían reflejar la realidad de la lengua española tal y como la usan y manejan sus hablantes nativos, así como el contexto sociolingüístico 
de aprendizaje; asimismo, sería muy beneficioso la contextualización y adaptación del MCER (Sossouvi, 2016). Además de una introducción temprana pero gradual de la lengua española en la vida académica de los aprendientes, el establecimiento de un dispositivo híbrido que combina sesiones presenciales y la educación a distancia (en colaboración con las universidades españolas o de países de habla española), una actualización lingüística y cultural de los profesores de ELE del país resulta imprescindible.

\section{Reflexiones finales}

La meta fundamental de este trabajo fue analizar las producciones orales espontáneas de estudiantes universitarios benineses de ELE e intentar caracterizarlas lingüísticamente. Para sintetizar en pocas líneas los hallazgos de este trabajo, se podría decir que los estudiantes participantes tienen dificultades para producir en la lengua española y que se apoyan en todo su bagaje lingüístico previo para comunicarse. Los datos empíricos analizados revelan algunos factores que predisponen a los errores, tales como el papel tanto del francés, de las lenguas nacionales u otras lenguas estudiadas, como del control incompleto de la lengua meta, la insuficiente exposición a la lengua meta, la metodología y los materiales con lenguaje no auténtico utilizados, e incluso el reflejo de su cultura lingüística. Además, evidencian la interacción, la mezcla y combinación de las lenguas disponibles contrastando con los hallazgos de Sossouvi (2004, 2009a; 2009b). Sugieren también que el fenómeno de influencia translingüística está presente en todos los niveles, del principio a los niveles más altos, y refleja la dinámica del contacto de lenguas. La teoría general sobre el proceso de influencia translingüística entre la lengua meta y las demás lenguas aprendidas queda por precisar, sobre todo cuando el aprendizaje tiene lugar en un contexto genuinamente multilingüe como el de África; ya que su realidad es tan amplia y compleja como el universo.

Las incorrecciones registradas reflejan los esfuerzos que están realizando para formular hipótesis sobre la lengua española, así como su cultura lingüística. Esta circunstancia exige, por consiguiente, que todos los actores de la enseñanza del español en Benín y todas las personas con capacidad de decisión, empiecen a analizar la realidad para así adaptar la enseñanza de esta lengua a la cultura lingüística de los aprendientes. Los hallazgos del presente estudio pueden ser de gran utilidad tanto para el profesorado de ELE, los diseñadores de materiales didácticos, los investigadores, como los formadores de traductores e intérpretes. Presentan una riqueza increíble que permite ilustrar un fenómeno frecuente y polémico: la mezcla de códigos, el préstamo y el mito de la pureza. 
Finalmente, quisiéramos volver a hacer constar que el presente estudio constituye, por nuestra parte, un primer acercamiento a la caracterización de las interacciones orales de los estudiantes universitarios benineses de ELE y podría servir, en un futuro cercano, como fundamento para una investigación más detallada y profunda de la cuestión. Somos conscientes de las limitaciones del trabajo que disminuyen la fiabilidad de los resultados obtenidos, tales como el análisis únicamente de los errores, la muestra limitada y el abanico de las producciones. Un mejor análisis requeriría de datos más extensos respecto a las producciones orales, incluyendo las escritas; la evaluación de la gravedad de los errores o el estudio de una sola categoría de errores problemáticos, e incluso desarrollar nuevas investigaciones incluyendo nuevas perspectivas, tales como el análisis del discurso y el de la conversación.

\section{References:}

1. Buck, J. \& McAllister, J. (2011). Mise en place d'un dispositif d'apprentissage hybride à l'université. Les Cahiers de l'Apliut, 30 (1), pp. 83-101. Recuperado de: http://apliut.revues.org/571. DOI: 10.4000/apliut.571.

2. Cenoz, J. (2003). The role of typology in the organization of the multilingual lexicon. En Cenoz, J., Hufeisen, B. \& Jessner, U. (Eds.), The multilingual lexicon (pp. 103-116). Dordrecht: Kluwer.

3. Cenoz, J., Hufeisen, B. \& Jessner, U. (eds.) (2001). Cross-linguistic Influence in Third Language Acquisition: Psycholinguistic Perspectives. Clevedon: Multilingual Matters.

4. Clyne, M. (2003). Dynamics of language contact. Cambridge: Cambridge University Press.

5. Coder, S. P. (1971). Idiosyncrasic dialects and error analysis. International Review of Applied Linguistics, 9(2), pp. 147-159.

6. De Angelis, G. (2007). Third or Additional Language Acquisition. Clevedon: Multilingual Matters.

7. Dewaele, J.-M. (1998). Lexical Inventions: French Interlanguage as L2 versus L3. Applied Linguistics, 19 (4), pp. 471-490.

8. Ellis, R. (2008). The study of second language acquisition. Oxford: Oxford University Press.

9. Field, Fr. W. (2002). Linguistic borrowing in bilingual contexts. Amsterdam / Filadelfia: John Benjamins.

10. Fuller, J. M. (1999). Between three languages: Composite structure in interlanguage. Applied Linguistics, 20(4), pp. 534-561.

11. González Alonso, J., Villegas, J. \& García Mayo, M. del P. (2016). English compound and non-compound processing in bilingual and 
multilingual speakers: Effects of dominance and sequential multilingualism. Second Language Research, 32, pp. 503-535.

12. Herdina Ph. \& Jessner, U. (2002). A Dynamic Model of Multilingualism: Perspectives on change in psycholinguistics. Clevedon: Multilingual Matters.

13. Jake, J. L. \& Myers-Scotton, C. (1997). Relating interlanguage to codeswitching: The Composite Matrix Language. Proceedings, the 1996 Boston University Conference on Language Development, pp. 319-330.

14. Jake, J. (1998). Constructing Interlanguage: Building a composite matrix language, Linguistics, 36(2), pp. 333-382.

15. James, C. (1990). Contrastive analysis. Harlow: Longman.

16. Jarvis, S. (2014). Influences of previously learned languages on the learning and use of additional languages. En Juan-Garau, M. \& Salazar-Noguera, J. (Eds.), Content-based Language Learning in Multilingual Educational Environments (pp. 69-86). Berlin: Springer.

17. Jarvis, S. \& Pavlenko, A. (2008). Crosslinguistic influence in language and cognition. Nueva York: Rutledge.

18. Jessner, U. \& Cenoz, J. (2007). Teaching English as third language. En Cummins, J. \& Davison, C. (Eds.), International Handbook of English Language Teaching (pp. 155-167). Nueva York: Springer.

19. Karim, Kh \& Nassaji, H. (2013). First language transfer in second language writing: An examination of current research. Iranian Journal of Language Teaching Research, 1(1), pp. 117-134.

20. Kiuchukova-Petrinska, B. (2010). De los errores pragmáticos de los aprendientes búlgaros de español. La pragmática en la enseñanza. Sofía: Editorial Universitaria San Clemente de Ójrid.

21. Lightbown, P \& Spada, N. (1993). How languages are learned. Oxford: Oxford University Press.

22. Major, R. C. (2001). Foreign accent: the ontogeny and phylogeny of second-language phonology. Mahwah/ NJ: Lawrence Erlbaum.

23. Meisel, J. (2007). Exploring the limits of the LAD. Working Papers in Multilingualism, 80, pp. 3-31.

24. Moattarian, A. (2013). Bidirectional Crosslinguistic Influence in Language Learning: Linguistic Aspects and Beyond. International Journal of Linguistics, 5(4), pp.38-49.

25. Montrul, S. (2008). Incomplete acquisition in bilingualism. Reexamining the age factor. Amsterdam: John Benjamins.

26. Muysken, P. (2000). Bilingual speech. A typology of code-mixing. Cambridge: Cambridge University Press. 
27. Myers-Scotton, C. M. \& Jake, J. L. (1995). Matching lemmas in a bilingual language production model: Evidence from intrasential codeswitching. Linguistics, 33(5), pp. 981-1024.

28. Myers-Scotton, C. M. \& Jake, J. L. (2000). Four types of morpheme: Evidence from aphasia, codeswitching, and second language acquisition. Linguistics, 38(6), pp. 1053-1100.

29. Nemser, W. (1971): Approximative systems of foreign language learners. International Review of Applied Linguistics, 9(2), pp. 115123.

30. Odlin, T. \& Jarvis, S. (2004). Same source, different outcomes: A study of Swedish influence on the acquisition of English in Finland. International Journal of Multilingualism, 1(2), pp. 123-140.

31. Pavlenko, A. \& Jarvis, S. (2002). Bidirectional transfer. Applied Linguistics, 23(2), pp. 190-214.

32. Pennycook, A. (2010). Language as a local practice. Londres: Routledge.

33. Porquier, R. (1986). Remarques sur les interlangues et leurs descriptions. Études de linguistique appliquée, 63, pp. 101-107.

34. Porquier, R. \& Frauenfelder, U. (1980). Enseignants et apprenants face à l'erreur ou de l'autre côté du miroir. Le français dans le monde, 154, pp.29-36.

35. Ramón Torrijos, M. del M. (2009). Effects of cross-linguistic influences on second language acquisition: A corpus based study of semantic transfer in written production. Revista de Lingüística y Lenguas Aplicadas, 4, pp. 147-159. Recuperado de: http://dx.doi.org/10.4995/rlyla.2009.741.

36. Selinker, L. (1972). Interlanguage. International Review of Applied Linguistics, 10(3), pp. 209-231.

37. Singleton, D. (1999. Exploring the second language lexicón. Cambridge: Cambridge University Press.

38. Sossouvi, L.-F. (2004). Análisis de errores de los alumnos benineses de español como lengua extranjera. (Tesis doctoral). Universidad de Alcalá, Facultad de Filosofías y Letras, España.

39. Sossouvi, L.-F. (2009a). El aprendizaje del español en la enseñanza secundaria de Benín: implicaciones teóricas. Estudios Filológicos, 44, pp. 211-225.

40. Sossouvi, L.-F. (2009b). La adquisición del español como lengua extranjera por aprendientes francófonos de África: Implicaciones teóricas y pedagógicas. Didáctica. Lengua y Literatura, 21, pp. 319344.

41. Sossouvi, L.-F. (2014). La lengua castellana en Benín: tendencias actuales después de seis décadas. En Serrano Avilés, J. (Ed.), La 
situación del español en África subsahariana (pp. 100-126), Madrid: Editorial Catarata.

42. Sossouvi, L.-F. (2016). Enseñanza y aprendizaje del ELE en Benín: ¿qué puede aportar la adaptación del MCER? Tejuelo Revista de Didáctica de la Lengua y la Literatura. Educación, 23, pp. 36-62.

43. Su, I-R. (2012). Bi-directional transfer in Chinese EFL learners' apologizing behavior. Studies in Linguistics, 38(2), pp. 237-266. 